\title{
1 Public Health: Zentrale Begriffe, Disziplinen und Handlungsfelder
}

\section{Matthias Egger, Oliver Razum}

In diesem einführenden Kapitel lernen wir die zentralen Begriffe, Disziplinen und Handlungsfelder von Public Health kennen. Ein Blick in das 19. Jh. zeigt, dass Public Health zu Beginn überraschenderweise weniger mit der Medizin als mit dem Ingenieurwesen zu tun hatte. Die Geschichte macht auch verständlich, warum heute der englische Begriff ,Public Health' auch im Deutschen gebräuchlich ist. Public Health und Medizin unterscheiden sich in ihrer Sicht auf Krankheit und Gesundheit. Anders als im medizinischen Denken steht in Public Health die Entstehung von Gesundheit (Salutogenese) und nicht die Entstehung von Krankheit (Pathogenese) im Mittelpunkt. Zu den Kernthemen von Public Health gehört u.a. die gesundheitliche Ungleichheit zwischen verschiedenen Bevölkerungsgruppen, z. B. die Ungleichheit im Zusammenhang mit der sozialen Schichtzugehörigkeit und dem Geschlecht. Bei vielen Public-Health-Fragen spielen auch ethische Aspekte eine Rolle. Während in der Medizinethik die Arzt-Patient-Beziehung im Mittelpunkt steht, ist es in der Public-Health-Ethik das Verhältnis zwischen den Institutionen und den BürgerInnen. Wir schließen das Kapitel mit einem kritischen Blick auf die Public Health Genomics und ihrem Versprechen einer individualisierten Prävention.

Schweizerische Lernziele: CPH 1-3, CPH 28-34

\subsection{Definition}

Unter Public Health verstehen wir eine von der Gesellschaft organisierte, gemeinsame Anstrengung, mit dem Ziel der

- Erhaltung und Förderung der Gesundheit der gesamten Bevölkerung oder von Teilen der Bevölkerung,

- Vermeidung von Krankheit und Invalidität,

- Versorgung der Bevölkerung mit präventiven, kurativen und rehabilitativen Diensten.

Im deutschsprachigen Raum wird synonym auch etwas umständlich von der öffentlichen Gesundheitspflege gesprochen. Der Begriff der Volksgesundheit ist durch den Nationalsozialismus schwer belastet (s. Kap. 1.2) und wird deshalb nicht verwendet. Aus den genannten Gründen ist der englische Begriff ,Public Health' auch im Deutschen gebräuchlich. Im Gegensatz zur kurativen Individualmedizin richtet Public Health den Blick auf die gesamte Bevölkerung oder auf Bevölkerungsgruppen und 
beschäftigt sich hier mit ethisch (s. Kap. 1.6) und ökonomisch (s. Kap. 2.5.1) vertretbaren Maßnahmen der Gesundheitsförderung, der Krankheitsprävention und der Versorgung.

Handlungsfelder von Public Health sind

- die wissenschaftliche Forschung an universitären Instituten: In Deutschland geschieht das v. a. an gesundheitswissenschaftlichen Instituten, in der Schweiz an Instituten für Sozial- und Präventivmedizin.

- die Praxis in den Public-Health-Institutionen: In der Schweiz sind hierfür z.B. die kantonalen Gesundheitsämter und das Bundesamt für Gesundheit zuständig, in Deutschland u.a. das Robert Koch-Institut.

- die Gesundheits- und Sozialpolitik, die durch Verordnungen und Gesetze das Gesundheitswesen steuert und gesundheitsfördernde Arbeits- und Lebensbedingungen schafft.

Zu den Aufgaben von Public-Health-Institutionen gehört es, die Gesundheit der Bevölkerung zu schützen und zu überwachen (Surveillance), etwa im Zusammenhang mit Infektionskrankheiten (s. Kap. 8.2.2), der Lebensmittelsicherheit (s. Kap. 5.1.4), der Sicherheit am Arbeitsplatz (s. Kap. 6.1.2) oder der Luftverschmutzung (s. Kap. 5.2). Darüber hinaus sind sie u.a. für die Erarbeitung und Durchführung von Impfprogrammen (s. Kap. 8.4.1), Screening-Programmen (s. Kap. 4.5.4) und Aufklärungskampagnen (s. Kap. 4.2.1) zuständig. Hierbei arbeiten Fachleute verschiedenster Disziplinen aktiv zusammen (s. Kap. 1.4). Beispiele für gesundheitspolitische Maßnahmen sind Rauchverbote in öffentlichen Räumen (s.a. Kap. 7.2.3) und die laufenden Bestrebungen, Gesundheitsförderung und Prävention in Deutschland und der Schweiz durch Präventionsgesetze umfassend zu stärken.

Der Master of Public Health (MPH) ist ein international anerkannter akademischer Grad, der im angelsächsischen Raum (z. B. an der geschichtsträchtigen London School of Hygiene \& Tropical Medicine oder an den Schools of Public Health nordamerikanischer Universitäten), aber auch an verschiedenen Hochschulen in Deutschland und der Schweiz erworben werden kann. Ein MPH-Studium ist in der Schweiz Teil der Weiterbildung zum Facharzt in Prävention und Gesundheitswesen. In Deutschland kann der Facharzt für Öffentliches Gesundheitswesen und der Facharzt für Hygiene und Umweltmedizin erworben werden. In beiden Ländern gibt es darüber hinaus einen Facharzttitel im Bereich der Arbeitsmedizin. 


\subsection{Geschichtliche Notizen}

Die soziale Frage

Die Entwicklung der modernen Public Health ist eng mit der sozialen Reformbewegung im 19. Jahrhundert verbunden, die darauf abzielte, die soziale Lage der Arbeiter und ihrer Familien zu verbessern. Abb. 1.1 illustriert die Lebensumstände der damaligen Arbeiterschaft am Beispiel einer Behausung in London. Angesichts dieser Zustände überrascht es nicht, dass London und andere europäische Städte zu jener Zeit immer wieder von Cholerapandemien heimgesucht wurden (s. a. Abb. 1.2) und dass dort auch die Tuberkulose grassierte. Im Zentrum der angestrebten Reformen standen die Verbesserung der sanitären Bedingungen in den Städten und der Verhältnisse am Arbeitsplatz. In England förderte der Public Health Act von 1848 den Bau von Wasserleitungen und Kanalisationsanlagen (s. a. Kap. 5.1.1). In Berlin trieb der Pathologe und Sozialreformer Rudolf Virchow (1821-1902, s. a. Kap. 2.1.1) den Bau von zentraler Wasserversorgung und Kanalisation voran, während in München Max von Pettenkofer (1818-1901) hierbei die treibende Kraft war.

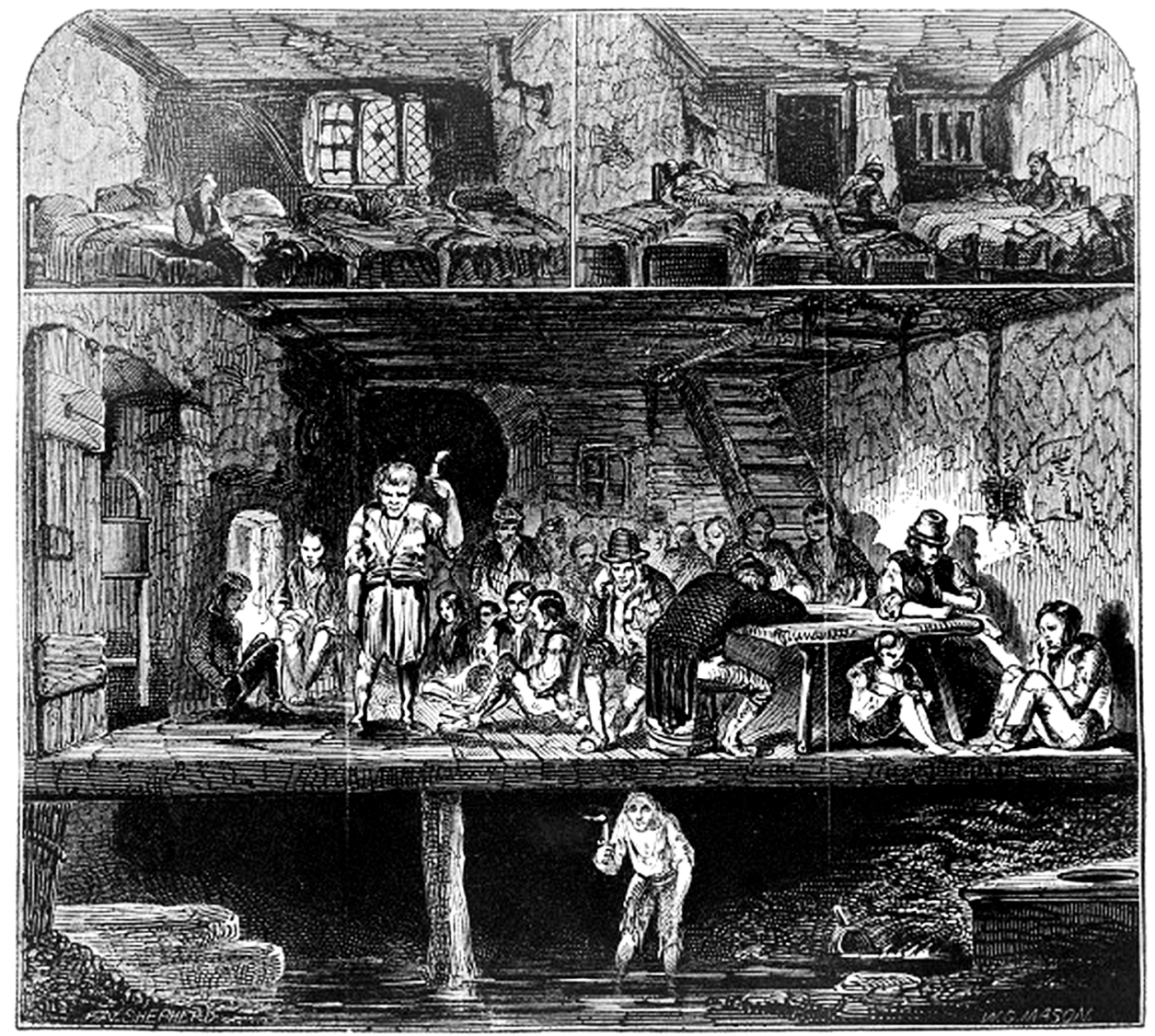

Abb. 1.1: Eine Londoner Behausung an der Themse im 19. Jahrhundert. Die Notdurft wurde am Fluss verrichtet (Quelle: Wellcome Images. http://images.wellcome.ac.uk/). 


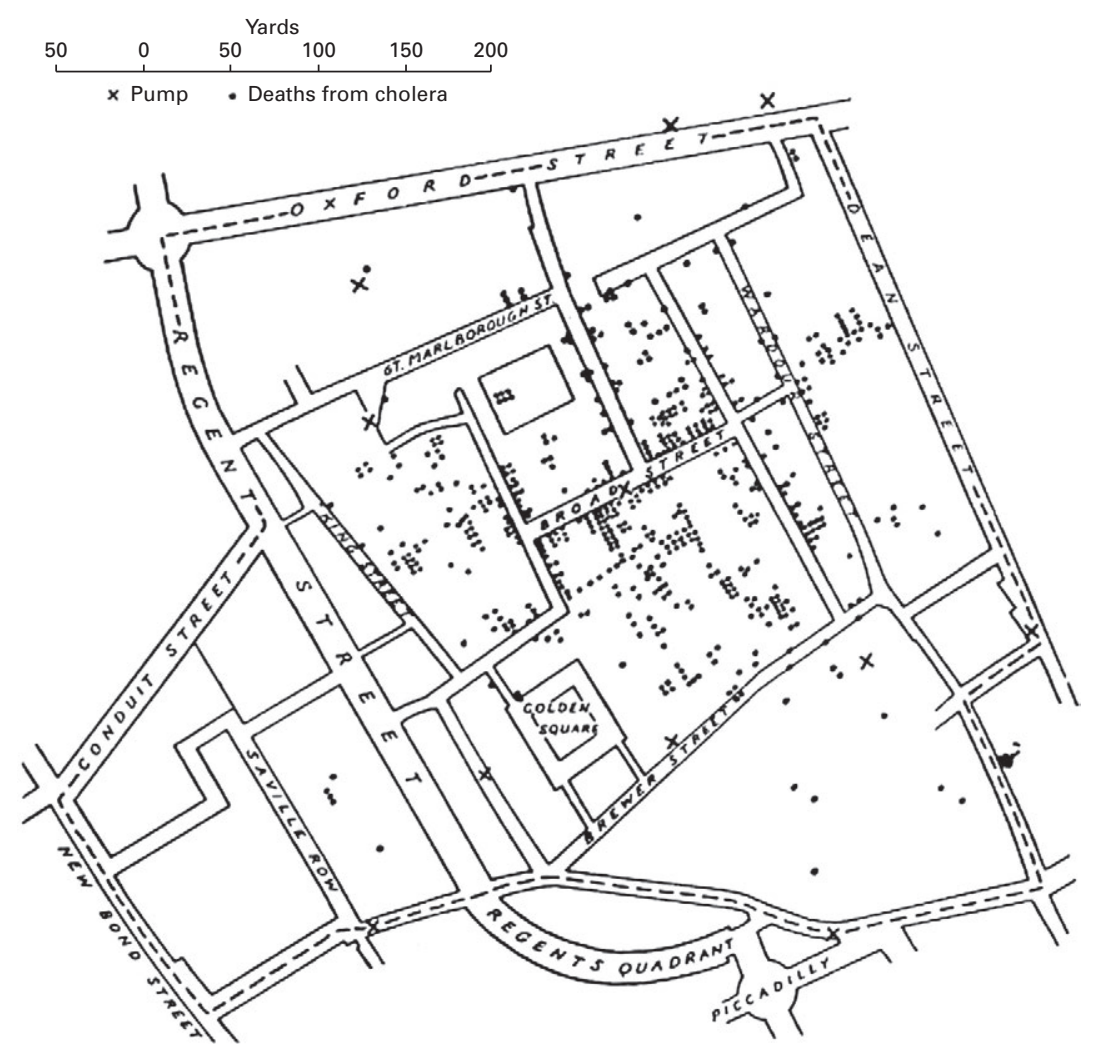

Abb. 1.2: Karte der Cholera-Todesfälle im Rahmen der Pandemie von 1854, die rund um die Broad Street-Wasserpumpe auftraten. Der Epidemiologe John Snow (1813-1858, s.a. Kap. 2.1.1) folgerte hieraus, dass die Cholera durch verschmutztes Trinkwasser übertragen wird und entfernte den Pumpengriff, um weitere Ansteckungen zu verhindern. (Rekonstruktion der Karte nach Snows Angaben durch den medizinischen Geografen E.W. Gilbert, 1958) (Quelle: Gilbert E W. Pioneer map and health and disease in England. Geographical Journal 1958; 124(2): 172-183).

\section{Hygiene und Soziallhygiene}

Pettenkofer hatte ab 1865 den ersten Lehrstuhl für Hygiene in Deutschland inne. Zentrale Themen dieses neuen medizinischen Fachgebietes waren die Verhütung von Krankheiten und die Förderung der Gesundheit der Bevölkerung. Pettenkofers besonderes Interesse galt dabei der physikalischen und chemischen Umwelt. Er gilt deshalb als Wegbereiter der Umweltepidemiologie und Umweltmedizin (s. Kap. 5). Mit der Entdeckung der Bakterien und dem im Jahr 1882 durch Robert Koch (1843-1910) erfolgten Nachweis, dass ein einziger, eindeutig identifizierbarer Krankheitserreger ( $M y$ cobacterium tuberculosis) die Tuberkulose in ihren verschiedenen Ausprägungen verursacht, wurde die Bakteriologie zur führenden Gesundheitswissenschaft des ausgehenden 19. Jahrhunderts. Damit war die Debatte um die Frage, wodurch Krankheiten verursacht werden, jedoch noch nicht abgeschlossen. Die von Alfred Grotjahn (18691931) begründete Sozialhygiene stellte die monokausale Erklärung der Entstehung von 


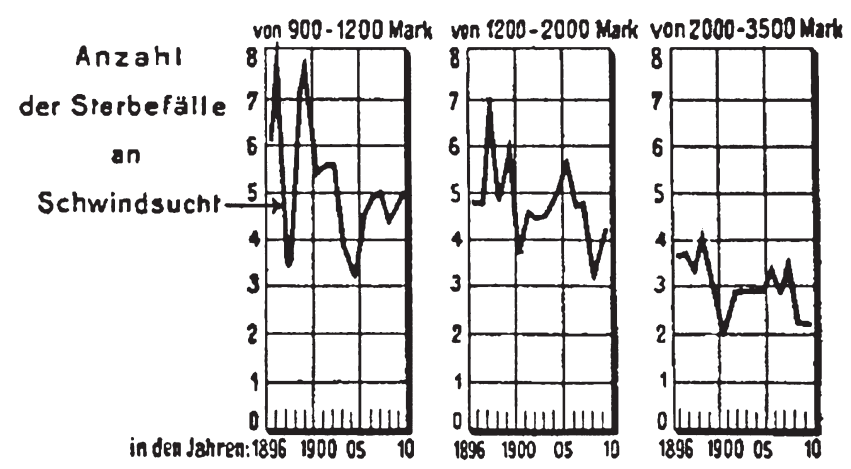

Abb. 1.3: Beispiel einer sozialhygienischen Studie, die zu Beginn des 20. Jh. in Deutschland durchgeführt wurde. Die Grafik zeigt das Einkommen (in Mark) und die Tuberkulosesterblichkeit (pro 1.000 Einwohner) in Hamburg in den Jahren zwischen 1896 und 1910. Menschen mit höherem Einkommen wiesen eine deutlich niedrigere Sterblichkeit an Tuberkulose auf als Menschen aus niedrigeren Einkommensschichten (Quelle: Mosse M, Tugendreich G. Krankheit und Soziale Lage, 1913).

Infektionskrankheiten in Frage und betonte die Wichtigkeit von gesellschaftlichen Einflüssen, wie z. B. von engen und unhygienischen Wohnverhältnissen, schlechter Ernährung oder niedrigem Einkommen auf die Krankheitsentstehung (s. Abb. 1.3 mit einem Beispiel einer Studie aus dieser Zeit). Grotjahn vertrat später allerdings als Mitglied der Gesellschaft für Rassenhygiene auch eugenische Vorstellungen (s.u.). Zu Beginn des 20. Jahrhunderts war Deutschland auf dem Gebiet der Hygiene führend, was sich u.a. daran zeigte, dass die erste Internationale Hygiene-Ausstellung 1911 in Dresden von mehr als fünf Mio. Menschen (!) besucht wurde. Die Schaffung von kommunalen Gesundheitsämtern in Deutschland (heute oft als Fachdienst Gesundheit bezeichnet) ist ein bleibender Verdienst jener Zeit.

\section{Eugenik und Nationalsozialismus}

Die Sozialhygiene war eng mit der Eugenik oder Rassenhygiene verbunden. Hierunter verstand man die Anwendung von Erkenntnissen aus der Humangenetik auf die Bevölkerung mit dem Ziel, die Fortpflanzung von "Gesunden“ zu fördern und dadurch den Anteil an Menschen mit „positiven“ Erbanlagen zu erhöhen. Auch der Sozialhygieniker Grotjahn (s.o.) war Mitglied der Gesellschaft für Rassenhygiene und befürwortete die Zwangssterilisierung von Menschen mit körperlicher oder geistiger Behinderung, von Menschen mit Epilepsie und von Alkoholkranken. Die Eugenik geht auf den englischen Naturforscher Francis Galton (1822-1911) zurück, einem Vetter Charles Darwins. Die ersten eugenisch motivierten Sterilisationen wurden in Europa bereits um 1890 durch den Psychiater und Ameisenforscher Auguste Forel (1848-1931) in der Psychiatrischen Universitätsklinik Burghölzli in Zürich durchgeführt. Nach ihrer Machtergreifung im Jahr 1933 setzten die Nationalsozialisten das auf den Ideen der Rassenhygiene beruhende, menschenverachtende Ziel eines „rassenreinen arischen Volkskörpers” konsequent und mit unglaublicher Grausamkeit mit Hilfe von Massensterilisierungen, Massentötungen und Genozid durch. Weniger bekannt sind andere Aspekte der nationalsozialistischen Gesundheitspolitik, wie Maßnahmen gegen das Rauchen, Verbote von 
petrochemischen Kanzerogenen und der Schutz vor Asbest am Arbeitsplatz. Tabak galt dabei nicht nur als Krebserreger und Ursache von Herzkrankheiten, sondern auch als Rassengift, das die Fruchtbarkeit und Arbeitskraft der Menschen einschränkt. Nichtrauchen war daher eine Gesundheitspflicht. Das Rauchen in der Öffentlichkeit sowie die Tabakwerbung wurden eingeschränkt oder verboten. Die Web-Abb. 1.2.1 auf unserer Lehrbuch-Homepage zeigt ein Werbeplakat aus einer Kampagne gegen das Rauchen aus dem Jahr 1941. Die unglückliche Verbindung mit dem Nationalsozialismus, die sich im englischen Sprachraum in Begriffen wie "nicoNazi" oder "health facism" niederschlägt, belastet die Tabakprävention noch heute. Wie wenig erfolgreich die nationalsozialistische Tabakpolitik jedoch war, zeigt sich u. a. dadurch, dass die "Amis" (d. h. die amerikanischen Zigaretten) Deutschland nach dem Zweiten Weltkrieg im Sturm eroberten.

\section{Neuere Entwicklungen und Herausforderungen}

Nach dem Zweiten Weltkrieg entwickelte sich in der Deutschen Demokratischen Republik (DDR; 1949-1990) ein zentralistisches Gesundheitssystem, das der Prävention, der Gesundheitserziehung und dem Gesundheitsschutz in den Betrieben eine große Bedeutung zuwies. Die Gesundheitssysteme in der Bundesrepublik Deutschland (BRD) und der Schweiz wurden hingegen dezentral und libertär organisiert (s.a. Kap. 3). Krankheit berechtigte hier zur selbstverantwortlichen Inanspruchnahme von gesetzlich verankerten, versicherten medizinischen Leistungen. Der salutogenetische Public-Health-Ansatz (s. Kap. 1.4.2) rückte dabei in den Hintergrund. Krankheit wurde zunehmend als medizinisch-technisches Problem verstanden, für das Fachärzte und Krankenhäuser zuständig waren. Parallel zum Anstieg der Lebenserwartung sank die Kinderzahl pro Familie, ebenso der Anteil der Erwerbstätigen im Verhältnis zu den Nichterwerbstätigen.

Diese demographische Entwicklung (s. a. Kap. 2.2) führte in den Industrienationen zu einer zunehmenden Alterung der Bevölkerung. Damit nahm auch die Häufigkeit chronisch-degenerativer Krankheiten, v. a. von Herz-Kreislauf-Erkrankungen und bösartigen Tumoren zu (s. Kap. 7.1 und Kap. 7.2). Gleichzeitig stieg die Anzahl der psychischen und psychosomatischen Erkrankungen (s. Kap. 7.7) an. Im Rahmen des Risikofaktorenmodells wurde nun nach biomedizinischen, aber auch nach psychosozialen Faktoren gesucht, die mit einer erhöhten Erkrankungswahrscheinlichkeit einhergehen. Man hoffte, hierdurch Strategien zur Prävention und Gesundheitsförderung entwickeln zu können (s. Kap. 4.2 und 4.3). Mit Hilfe der 1948 gestarteten Framingham Studie, eine Kohortenstudie (s. a. Kap. 2.1.5) der Bevölkerung der Stadt Framingham im US-Bundesstaat Massachusetts, wurden z.B. verschiedene Risikofaktoren identifiziert, die zur Entstehung von Herzinfarkt und Schlaganfall beitragen (s.a. Kap. 7.1). In der Folgezeit wurden die Gesundheitswissenschaften zunehmend interdisziplinär und multiprofessionell (s. Kap. 1.4). Man wandte sich nun auch neuen Feldern zu, wie etwa der Evaluation und der Kosten-Nutzen-Bewertung medizinischer Maßnahmen (klinische Epidemiologie und Gesundheitsökonomie, s. a. Kap. 2.1.7 und Kap. 2.5), der Versorgung der Bevölkerung und der Steuerung der Gesundheitssysteme (Versorgungs- und Gesundheitssystemforschung, s.a. Kap. 3) und den Herausforderungen auf globaler Ebene (Global Health, s. a. Kap. 9).

Die demographische Entwicklung (s. Kap. 2.2), die Zunahme von Übergewicht und chronisch-degenerativen Erkrankungen (s.a. Kap. 7) sowie die sozialen Ungleichheiten 
in Gesundheitszustand und Versorgung (s.a. Kap. 1.3.1 und Kap. 3) sind wichtige Felder, auf denen Public Health schon heute stark gefordert ist. Darüber hinaus gibt es noch einige Hindernisse, die es in den kommenden Jahren zu überwinden gilt. Hierzu gehören die dominante Rolle der kurativen Medizin in den fragmentierten Gesundheitssystemen (s.a. Kap. 3), die oft lückenhafte Zusammenarbeit zwischen den Gesundheitswissenschaften und den Einrichtungen des öffentlichen Gesundheitsdienstes sowie die fehlende Ausrichtung der Forschung auf die konkreten Fragestellungen der öffentlichen Gesundheitspolitik (s. Kap. 1.6).

\subsection{Zentrale Konzepte und Themen}

\subsubsection{Gesundheit und Krankheit}

Ein Mensch ist nicht einfach entweder krank oder gesund. Die Betrachtungsweise kann sich schon durch die eingenommene Perspektive ändern: Eine Ärztin diagnostiziert bei einem Menschen eine Vorstufe von Krebs, der Betroffene verspürt jedoch noch keine Symptome und fühlt sich gesund. Auch handelt es sich bei Gesundheit und Krankheit nicht um ein Phänomen, das nur zwei Zustände einnehmen kann. Zwischen "krank" und "gesund" können zahlreiche Zwischenstufen bestehen. Zudem gibt es sehr unterschiedliche Vorstellungen darüber, wie Krankheit und Gesundheit entstehen. Krankheit und Gesundheit lassen sich darüber hinaus auch auf unterschiedlichen Ebenen betrachten, auf der des Individuums (dies ist v. a. die Sichtweise der Medizin) und auf der der Bevölkerung (dies entspricht der Perspektive von Public Health). Public Health und Medizin unterscheiden sich damit jedoch nicht nur in ihren Sichtweisen, sondern auch in den von ihnen gewählten Strategien, um Gesundheit zu erhalten, zu verbessern oder wiederherzustellen.

Es gibt zahlreiche Konzepte und Modelle, die z.T. sehr unterschiedliche Sichtweisen auf Krankheit und Gesundheit erlauben. Exemplarisch werden hier die Konzepte der Pathogenese und der Salutogenese vorgestellt.

\section{Pathogenese}

Mit dem Begriff der "Pathogenese“ bezeichnet man die Entstehung und Entwicklung einer Krankheit. Pathogenetische Konzepte beschäftigen sich mit Prozessen, die zu Krankheiten führen und untersuchen mögliche Risikofaktoren für die Entstehung von Krankheiten. Sie schauen dabei in erster Linie auf Veränderungen, die sich an Organen, Gewebe und Zellen zeigen. Pathogenetische Konzepte bilden die Grundlage der naturwissenschaftlichen Medizin. Man kann sie auch als „Krankheitsmodelle" verstehen und damit den weiter unten beschriebenen "Gesundheitsmodellen" gegenüberstellen, derer sich Public Health häufig bedient.

Das biomedizinische Krankheitsmodell ist stark pathogenetisch geprägt. Es interpretiert Krankheit als eine Abweichung vom Normalzustand des Körpers. Krankheiten haben hier spezifische Ursachen (z. B. Bakterien). ÄrztInnen identifizieren diese Ursachen und können dann eine kausale ${ }^{1}$ - anstatt einer symptomatischen ${ }^{2}$ - Behandlung durch-

\footnotetext{
1 kausal: ursächlich

2 symptomatisch: an den Symptomen orientiert
} 
führen. Dieses stark naturwissenschaftlich beeinflusste Krankheitsmodell hat sich bei vielen Erkrankungen als sehr erfolgreich erwiesen. Daher werden u. a. in Deutschland und der Schweiz erhebliche Ressourcen in die Weiterentwicklung der Biomedizin investiert. Ein Schwerpunkt ist derzeit z. B. die Genomik (s. Kap. 1.7). In den vergangenen Jahrzehnten hat sich aber auch gezeigt, dass das biomedizinische Krankheitsmodell erhebliche Defizite aufweist. So geht es nicht ausreichend auf das individuelle Verhalten der Menschen ein, das insbesondere bei der Entstehung der immer bedeutsamer werdenden chronischen, nichtübertragbaren Krankheiten eine große Rolle spielt. Dieser Mangel wird durch das - ebenfalls stark pathogenetisch geprägte - Risikofaktorenmodell zumindest ansatzweise behoben (s. Kap. 2.1.1). Darüber hinaus bleiben gesellschaftliche Determinanten von Gesundheit und Krankheit nahezu unberücksichtigt. Dies ist in hohem Maße unbefriedigend, da u. a. sozioökonomische Benachteiligungen bei der Entstehung von Krankheit unzweifelhaft eine bedeutende Rolle spielen (s. Kap. 1.3.2). Weiterhin vermag das biomedizinische Krankheitsmodell nicht zu erklären, warum bestimmte Menschen gesund bleiben und wie sich Gesundheit fördern lässt. Spätestens hier zeigen sich die Stärken eines salutogenetischen Modells.

\section{Salutogenese}

Das Wort „Salutogenese“ bezeichnet analog dem Wort „Pathogenese“ die Entwicklung und Entstehung von Gesundheit. Es wurde in den 1970er-Jahren von Aaron Antonovsky im Rahmen seines salutogenetischen Modells geprägt (Näheres zu Antonovsky in den Internetquellen auf unserer Lehrbuch-Homepage). Anders als im medizinischen Denken steht hierbei die Gesundheit und nicht die Krankheit im Mittelpunkt. Antonovsky unterscheidet nicht zwischen zwei sich ausschließenden Begriffen "gesund" und "krank". Vielmehr interpretiert er Gesundheit und Krankheit als Endpunkte einer Linie. Zwischen diesen Endpunkten liegt ein Kontinuum von zahlreichen möglichen Zwischenstufen. Im Laufe des Lebens verändert sich der Gesundheitszustand eines Menschen auf diesem Kontinuum ständig. Antonovsky betrachtet Krankheiten somit als einen normalen Teil des menschlichen Lebens. Gesundheit ist also nicht der Regelfall und Krankheit nicht lediglich eine Abweichung von einem normalerweise bestehenden Gleichgewicht (Homöostase). Antonovsky spricht in seinem Modell der Salutogenese von einem Zustand der Heterostase, in dem sich der Mensch befindet. Er betont damit die ständigen Veränderungen, denen der Organismus infolge der Einwirkung äußerer Stressoren ausgesetzt ist. Nur durch ständige aktive Anpassungsleistungen und Auseinandersetzungen mit solchen Stressoren bleiben Menschen gesund.

Die beiden äußeren Punkte des von ihm beschriebenen Kontinuums bezeichnet Antonovsky als "health-ease" (Gesundheit) und "dis-ease“ (Krankheit). Hiervon leitet er die Bezeichnung HEDE-Kontinuum ab. Aus salutogenetischer Sicht soll ein Mensch stets aktiv danach streben, auf diesem Kontinuum möglichst nahe an den Punkt "Gesundheit" zu gelangen. Widerstandsressourcen helfen ihm dabei, Stressoren zu überwinden und sich somit auf dem HEDE-Kontinuum in Richtung Gesundheit zu bewegen. Solche Ressourcen können zum einen auf der gesellschaftlichen Ebene liegen (hierzu gehört z. B. ein intaktes gesellschaftliches Umfeld). Zum anderen verfügt jeder Mensch aber auch in unterschiedlichem Ausmaß über individuelle Ressourcen, etwa bei der Problemlösefähigkeit ( $\rightarrow$ Kognition), beim Selbstvertrauen ( $\rightarrow$ Psyche), bei der durch Training erworbene Ausdauer ( $\rightarrow$ Körper) oder in finanzieller Hinsicht ( $\rightarrow$ Ökonomie). 
Hat ein Mensch belastende Situationen wiederholt erfolgreich bewältigt, kann sich bei ihm ein zunehmendes Kohärenzgefühl einstellen (auch im Deutschen wird hierfür häufig der englische Begriff Sense Of Coherence, SOC, benutzt). Menschen mit einem ausgeprägten Kohärenzgefühl sind dadurch in der Lage, mit Stressoren erfolgreich zu umzugehen oder diese sogar als positive Herausforderung zu erleben. Angemessene Bewältigungsstrategien (Coping-Strategien) wirken sich in Verbindung mit einem starken Kohärenzgefühl förderlich auf die Gesundheit aus. Viele Public-Health-Strategien zur Förderung der Gesundheit in der Bevölkerung zielen daher darauf ab, gesellschaftliche und individuelle Ressourcen zu stärken. Dieser Ansatz unterscheidet sich damit substanziell von der Betrachtungsweise der Medizin, die einen pathogenetischen Ansatz vertritt.

Neben dem beschriebenen Modell der Salutogenese gibt es noch weitere Konzepte, die die Gesundheit (anstatt der Krankheit) in den Mittelpunkt der Betrachtung stellen, etwa das Modell der Resilienz (siehe Internet-Ressourcen).

\subsubsection{Gesundheitliche Ungleichheiten}

Eines der Kernthemen von Public Health sind die Ungleichheiten zwischen verschiedenen Bevölkerungsgruppen in Bezug auf ihre Gesundheit. Die vorhandenen Unterschiede im Hinblick auf soziale Schicht, Region, Ethnie, Nationalität, Alter und Geschlecht gehen oft mit gesundheitlichen Ungleichheiten einher. Diese Ungleichheiten betreffen neben dem Gesundheitszustand und den Gesundheitschancen (s.a. Kap. 4.1) auch das Gesundheitsverhalten und den Lebensstil (s.a. Kap. 4.4) sowie den Zugang und die Inanspruchnahme von Leistungen des Gesundheitssystems (s. a. Kap. 3.2). Sie sind in der Regel nicht durch unterschiedliche Bedürfnisse der Menschen gerechtfertigt, sondern entstehen aufgrund von Privilegien oder Benachteiligungen. In diesem Abschnitt diskutieren wir stellvertretend hierfür die Ungleichheiten, die im Zusammenhang mit der sozialen Schichtzugehörigkeit und dem Geschlecht auftreten. Selbstverständlich bestehen oft gleichzeitig auch andere Formen der gesundheitlichen Ungleichheit, z.B. infolge des Alters oder der ethnischen Zugehörigkeit.

\section{Soziale Ungleichheit und Gesellschaft}

Sowohl in reichen wie auch in armen Ländern gibt es innerhalb von Gesellschaften oft große Unterschiede zwischen den Menschen hinsichtlich bestimmter Merkmale wie Einkommen, beruflicher Position, Bildung und Sozialprestige. Diese Merkmale bilden die Grundlage für die Eingruppierung der Menschen in soziale Schichten. Entsprechend der unterschiedlichen Ausstattung haben die Menschen in den verschiedenen sozialen Schichten unterschiedliche Chancen, nicht nur in gesellschaftlicher Hinsicht, sondern - damit einhergehend - auch bezüglich ihrer Gesundheit. Es sind also nicht nur Krankheitserreger oder individuelle gesundheitsschädigende Verhaltensweisen, die zu Erkrankungen führen. Vielmehr trägt auch die Ungleichverteilung von Ressourcen zu einem höheren Krankheitsrisiko in den benachteiligten Schichten bei.

Ungleichheit und Ungerechtigkeit: Das Wort "Ungleichheit“ (Inequality) bezeichnet zunächst nur Unterschiede in den gesundheitlichen Chancen von Bevölkerungsgruppen. Solche Unterschiede kommen häufig vor. Oftmals sind sie nicht zu ändern oder 
werden sogar freiwillig von den Betroffenen hervorgerufen. So haben z. B. ältere Menschen ein höheres Risiko zu versterben als jüngere - daran ist leider nichts zu ändern. Ein anderes Beispiel sind Mountainbiker, die ein höheres Verletzungsrisiko als Menschen haben, die keinen Sport treiben. Dieses zusätzliche Risiko gehen sie aber freiwillig ein. Gleichzeitig ziehen sie möglicherweise auch gesundheitliche Vorteile aus dieser Tätigkeit.

Zahlreiche sozial bedingte gesundheitliche Unterschiede zwischen den Bevölkerungsgruppen sind jedoch vermeidbar und vor allem so gravierend, dass sie nicht einfach hingenommen werden können. Man spricht dann von Ungerechtigkeit (Inequity). So sind MigrantInnen in Deutschland eine sozial benachteiligte Gruppe. Sie weisen eine zwei- bis dreimal so hohe Säuglingssterblichkeit (Todesfälle im ersten Lebensjahr pro 1.000 Lebendgeborene) wie die nicht migrierte Mehrheitsbevölkerung auf. Todesfälle bei Säuglingen sind schwerwiegende Vorfälle, die vielfach vermeidbar sind. Um sie zu verhindern, müssen Schwangerenvorsorge, Geburtshilfe und kinderärztliche Versorgung für alle in gleicher Weise zugänglich sein und in gleich hoher Qualität angeboten werden. Menschen mit geringerer Bildung oder mit Problemen mit der deutschen Sprache nehmen jedoch Leistungen oft zu spät in Anspruch oder seltener als der Durchschnitt der Bevölkerung. Der Zugang zu Gesundheitseinrichtungen ist damit von der sozialen Lage abhängt, in der sich ein Mensch befindet. Wenn also Unterschiede in der Säuglingssterblichkeit zwischen den sozialen Schichten auftreten, so handelt es sich dabei um eine gesundheitliche Ungerechtigkeit.

Solche gesundheitlichen Ungerechtigkeiten treten oft in noch stärkerem Maße zwischen armen und reichen Ländern auf. Tabelle 9.2 in Kap. 9 zeigt dies eindrücklich am Beispiel der Säuglingssterblichkeit. Sie ist in Malawi 16-mal so hoch wie in der Schweiz oder in Deutschland. Die Gründe für diese Unterschiede sind nahe liegend. Malawi ist ein Land mit einem sehr niedrigem Entwicklungsstand (Least developed country, siehe Tab. 9.1) und sehr niedrigem pro-Kopf-Einkommen, während die Schweiz und Deutschland zu den High-income-Ländern gehören.

Die Whitehall-Studie: Die soziale und wirtschaftliche Lage von Menschen nimmt also großen Einfluss auf ihre Gesundheitschancen. Diese Erkenntnis gilt in den Industrienationen in ähnlicher Weise auch für chronische Erkrankungen (z. B. für Herz-KreislaufKrankheiten). Dies haben sozialepidemiologische Studien seit den 1960er-Jahren eindrücklich belegt. Am bekanntesten ist hier die Whitehall-Studie, die 1967 startete und nach dem Regierungsgebäude Whitehall in London benannt wurde. An dieser Kohortenstudie (s. Kap. 2.1) nahmen 18.000 männliche Angestellte der britischen Regierung teil. Die Studienergebnisse zeigten, dass Männer, die in der niedrigsten Job-Kategorie arbeiteten, eine höhere Sterblichkeit im Hinblick auf nahezu alle wichtigen Todesursachen hatten als Männer in der höchsten Job-Kategorie. Dies lag nicht etwa nur daran, dass schlechter gestellte Menschen meist auch ungesünder leben. Der Gradient blieb auch dann bestehen, wenn man die Unterschiede in der Prävalenz (Häufigkeit) von Risikofaktoren wie Rauchen statistisch ausglich (adjustierte). Zugespitzt formuliert: Pförtner haben hiernach ein höheres Risiko, einen Herzinfarkt zu erleiden oder frühzeitig zu versterben als leitende Angestellte. Der Herzinfarkt ist damit nicht eine Krankheit der Manager und Chefs, sondern der Gemanagten - der Menschen, die ein geringes Einkommen, einen niedrigen Bildungsstand und geringe Gestaltungsmöglichkeiten in ihrem Leben haben. Mittlerweile liegen aus Deutschland und der Schweiz ähnliche 


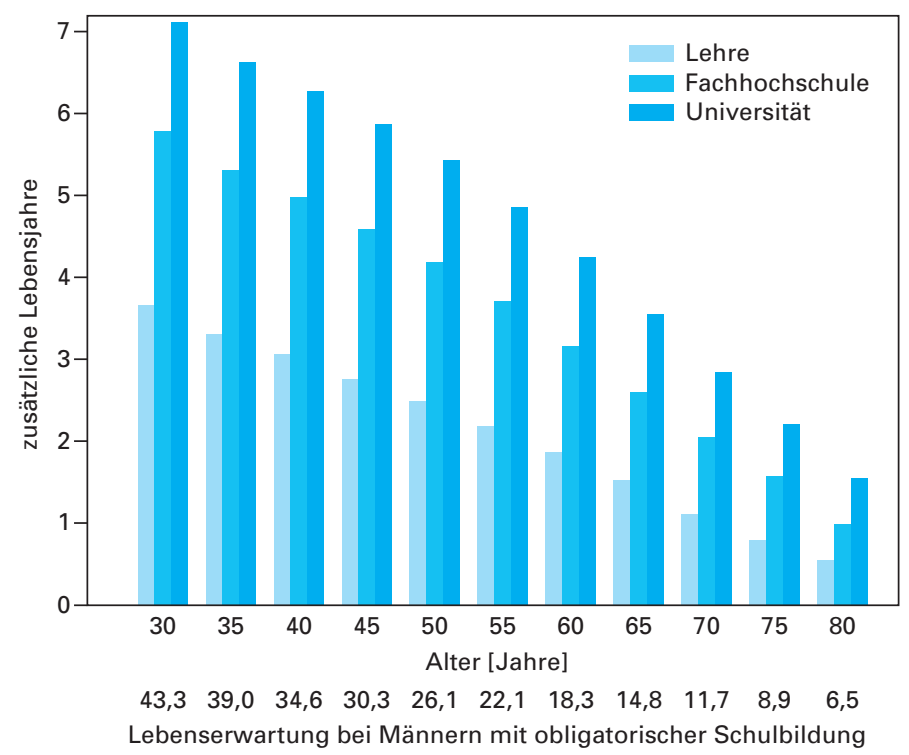

Abb. 1.4: Die weitere Lebenserwartung bei Männern in der Schweiz in Abhängigkeit von Alter und Bildung. Die Zahlen der unteren Zeile geben die Lebenserwartung bei Männern mit obligatorischer Schulbildung (Primarschule) an. Die Balken darüber zeigen die zusätzliche Anzahl an Lebensjahren bei Männern mit einer Berufsbildung in einer Firma (Lehre) und bei Männern mit einem Diplom einer Fachhochschule bzw. Universität. (Quelle: Spörri et al. Swiss Medical Weekly, 2006).

Beobachtungen vor. Abb. 1.4 aus der Schweiz zeigt die weitere Lebenserwartung von Männern verschiedener Altersgruppen und unterschiedlicher Bildung. Als Vergleichsgruppe dienen Männer, die lediglich die obligatorische Schulbildung durchlaufen haben. In allen Altersgruppen haben die Männer eine umso höhere weitere Lebenserwartung, je höher ihr Bildungsgrad ist.

Seit 1985 wird eine ähnliche Kohortenstudie wie die oben beschriebene WhitehallStudie unter dem Namen Whitehall I/ durchgeführt, nun auch mit weiblichen Teilnehmern. Sie versucht zu klären, wie der gesundheitliche Gradient in Abhängigkeit von der beruflichen Stellung oder der sozialen Lage entsteht. Die bisher vorhandenen Ergebnisse legen nahe, dass Stress hierbei eine große Rolle spielt und dass es verschiedene Arten von Stress gibt. Zur gesundheitlichen Ungleichheit trägt v. a. eine Form des Stresses bei, die entsteht, wenn den Betroffenen die Möglichkeit fehlt, ihr Leben oder ihre Arbeit selbst zu gestalten (s.a. Kap. 4.4.2, Kap. 6.3.1 und Kap. 7.1.2). Mittlerweile haben weitere Studien auch andere wichtige Faktoren identifiziert. So haben sozial schlechter gestellte Menschen oft einen schlechteren Zugang zu den Gesundheitsdiensten - dies gilt beispielsweise für MigrantInnen in Deutschland (s.o.). Auch gibt es Hinweise darauf, dass bereits während der Schwangerschaft und in der frühen Kindheit Gesundheitsrisiken „programmiert" werden (vgl. Kap. 1.7). Wer unter ungünstigen Bedingungen aufwächst, hätte demnach im späteren Leben ein höheres Erkrankungsrisiko als Menschen, die in wirtschaftlich entspannten Verhältnissen zur Welt kommen. Natürlich spielt hier auch eine Rolle, dass gesundheitsschädliche Verhaltensweisen unter 
sozial benachteiligten Menschen häufiger vorkommen. Darüber hinaus können Menschen, die wegen ihrer sozialen Benachteiligung ein höheres Krankheitsrisiko haben, dadurch auch sozial weiter absteigen, was wiederum das Krankheitsrisiko erhöht - ein Teufelskreis aus Krankheit und Armut.

Interventionen zur Verbesserung der Gesundheit auf der Bevölkerungsebene: Die heute vorliegenden Erkenntnisse aus der Sozialepidemiologie bestätigten nachdrücklich, dass es nicht nur individuelle, medizinische Risikofaktoren gibt, die unsere Gesundheit beeinflussen, sondern auch gesellschaftlich bedingte krankmachende oder schützende Faktoren - auch in der Schweiz und in Deutschland. Um die Gesundheit der Bevölkerung zu verbessern, reichen daher medizinische Maßnahmen allein nicht aus. Vielmehr ist es erforderlich, die Lebensbedingungen benachteiligter Gruppen zu verbessern. Auch müssen diese Gruppen gezielt angesprochen werden, um ihr gesundheitliches Wissen und ihren Zugang zu Gesundheitsdiensten zu verbessern. Die AutorInnen eines Berichts der Weltgesundheitsorganisation WHO, der unter dem Namen The Social Determinants of Health (Die sozialen Determinanten von Gesundheit) vorgelegt wurde, kommen darüber hinaus noch zu dem Schluss, dass hierzu endlich die Ungleichverteilung gesellschaftlicher Ressourcen (v. a. der finanziellen Mittel und des politischen Einflusses) beseitigt werden muss.

\section{Ungleichheit zwischen den Geschlechtern}

Das Geschlecht gehört zu den wichtigsten Determinanten gesundheitlicher Ungleichheiten. Im Englischen werden die kleineren und größeren Unterschiede zwischen Frauen und Männern vereinfachend in zwei Kategorien unterteilt:

- Der Begriff „Sex“ bezeichnet die biologischen (u.a. genetischen, anatomischen, physiologischen, immunologischen) Unterschiede zwischen den Geschlechtern. Das biologische Geschlecht beeinflusst z. B.

- die Wahrscheinlichkeit geboren zu werden: Das natürliche Verhältnis von Jungen zu Mädchen liegt hier bei 1,05 (105 Jungen : 100 Mädchen).

- das Risiko an bestimmten Erkrankungen zu leiden: So kommen z. B. Autoimmunerkrankungen bei Frauen häufiger vor als bei Männern.

- die Ausprägung von bestimmten Symptomen: Die beim Herzinfarkt auftretenden Symptome unterscheiden sich z. B. in Abhängigkeit vom Geschlecht der Betroffenen.

- die Behandlungsresultate: Frauen bilden z. B. nach Impfungen weniger Antikörper.

- die Lebenserwartung: In den Industrienationen liegt sie bei Frauen um etwa 5 Jahre höher als bei Männern (s.a. Kap. 2.2.4).

- Im Gegensatz hierzu beschreibt der Begriff „Gender" die psychologischen, sozialen und kulturellen Dimensionen des Geschlechts. Damit sind insbesondere die sozialen Rollen, Beziehungen, Verhaltensweisen und die Wertschätzung gemeint, die Frauen und Männern in einer Gesellschaft zugeschrieben werden. In Gesellschaften mit Bevorzugung männlicher Nachkommen, z. B. in Indien oder China, leistet die pränatale Bestimmung des Geschlechts mit Hilfe von Ultraschalluntersuchungen der selektiven Abtreibung von weiblichen Embryonen Vorschub. Obwohl es in Indien verboten ist, den Eltern das Geschlecht ihres Kindes während der Schwangerschaft 
mitzuteilen, wurden bei der Volkszählung im Jahre 2011 in Indien pro 1.000 Jungen unter sechs Jahren nur noch 914 gleichaltrige Mädchen registriert (Verhältnis 1,09).

Ein Beispiel für die Gesundheitsrelevanz der Geschlechterrollen ist die alkoholassoziierte Sterblichkeit: Das Trinken von Alkohol in großen Mengen ist in vielen Ländern Teil des männlichen Rollenverständnisses. So ist in Russland das „Zapoi“, ein mehrtägiger Alkoholexzess, ein lebensgefährliches Ritual unter den Männern. Es erstaunt deshalb nicht, dass Alkohol weltweit bei Männern für einen deutlich höheren Anteil an verlorenen Lebensjahren (Disability Adjusted Life Years) verantwortlich ist als bei Frauen (s.a. Kap. 9.1).

Die Förderung der Chancengleichheit von Männern und Frauen ist ein wichtiges Ziel der Politik. Sie gehört darüber hinaus zu den Millennium-Entwicklungszielen der Vereinten Nationen (Millennium Development Goals, s. a. Kap. 9.3.1). Der Europarat hat in diesem Zusammenhang den Begriff "Gender Mainstreaming" für eine gleichstellungsorientierte Politik auf allen Ebenen geprägt. Für Public Health und die Gesundheitspolitik bedeutet dies, dass die gesundheitliche Situation von Frauen und Männern in allen Bereichen der öffentlichen Gesundheit berücksichtigt werden soll. Auch die Versorgung soll verstärkt auf die spezifischen Bedürfnisse von Frauen und Männern ausgerichtet werden. Weiterhin sollen Public-Health-Maßnahmen und -Programme bezüglich ihrer Auswirkungen auf Geschlechterungleichheiten überprüft werden, und auch in der Gesundheitsberichterstattung soll die Geschlechterperspektive Berücksichtigung finden. Dies alles soll jedoch nicht isoliert geschehen, sondern unter Berücksichtigung anderer Dimensionen von Ungleichheit - wie z.B. von Ungleichheit, die durch die sozio-ökonomische Lage, das Alter oder den Migrationshintergrund hervorgerufen wird.

\subsection{Die Disziplinen der Public Health}

Unter dem Dach von Public Health wirken Disziplinen zusammen, die aus zwei unterschiedlichen wissenschaftlichen Traditionen kommen, der medizinisch-naturwissenschaftlichen und der sozial- und verhaltenswissenschaftlichen Tradition. Methodische Kernbereiche des interdisziplinären Fachs Public Health sind die Epidemiologie (s. Kap. 2.1) - ergänzt durch die Demographie (Kap. 2.2) und die Biostatistik (Kap. 2.3) - sowie die Sozialwissenschaften (s. Kap. 2.4) und die Gesundheitsökonomie (s. Kap. 2.5). Während die Epidemiologie quantitativ arbeitet, kommen in den Sozialwissenschaften sowohl quantitative als auch qualitative Methoden und Instrumente zum Einsatz.

Wichtige Einzeldisziplinen von Public Health sind:

- Sozialmedizin: Hierunter versteht man den Bereich der Medizin, der Zusammenhänge zwischen gesellschaftlichen Faktoren wie Einkommen oder Berufstätigkeit und gesundheitlichen Outcomes wie Erkrankung oder Tod untersucht (s.u. a. Kap. 4.4). Es bestehen enge Beziehungen zur Arbeitsmedizin (s. Kap. 6), deren Ziel es ist, arbeitsbedingte Erkrankungen u.a. durch Vorsorgemaßnahmen zu verhindern oder diese abzumildern.

- Medizinsoziologie: Sie untersucht ähnliche Fragen wie die Sozialmedizin und die Gesundheitspolitik, jedoch aus der Sicht der Soziologie. 
- Umweltmedizin: Die Umweltmedizin beschäftigt sich mit den Einflüssen von Umweltnoxen wie Lärm oder Luftschadstoffen auf die Gesundheit der Bevölkerung (s. Kap. 5).

- Präventivmedizin: Sie befasst sich mit der Krankheitsvorsorge und der Verhütung von Krankheiten, u. a. durch Prävention oder Früherkennung. Beispiele hierfür sind Impfungen zur Kontrolle von Infektionskrankheiten (Communicable Diseases; s. Kap. 8) und Screening-Tests zur Früherkennung von chronischen Krankheiten wie HerzKreislauf- oder Krebserkrankungen (Non Communicable Diseases; s. Kap. 7 sowie Kap. 4.5 und Kap. 4.6).

- Gesundheitspsychologie und -pädagogik: Diese Disziplinen untersuchen das menschliche Erleben und Verhalten bzw. deren Veränderungen. Ihre Erkenntnisse werden u. a. im Bereich der Gesundheitsförderung angewandt (s. Kap. 4.4.1 und Kap. 4.4.2).

- Gesundheitspolitik: Sie beschäftigt sich damit, wie Institutionen im Gesundheitssystem entstehen und wie diese arbeiten, wie dort Prozesse ablaufen und Entscheidungen getroffen werden (s. Kap. 3).

- Gesundheitsökonomie. Die Gesundheitsökonomie (s. Kap. 2.5) untersucht alle wirtschaftlichen Aspekte von Gesundheit, Krankheit und Gesundheitsversorgung.

- Organisations- und Managementwissenschaften: Sie befassen sich mit Prozessabläufen und Entscheidungsfindungen innerhalb von Institutionen, wie z. B. von Krankenhäusern.

- Ethik: Die Ethik beschäftigt sich mit gutem, richtigem und gerechtem menschlichen Handeln - ein Aspekt, der z. B. bei der Verteilung knapper Ressourcen eine große Rolle spielt (s. Kap. 1.6).

Die hier aufgelisteten Einzeldisziplinen lassen sich hinsichtlich ihres jeweiligen Forschungsgegenstands nicht immer eindeutig voneinander trennen. Sehr deutlich wird das in den Bereichen Sozialmedizin und Medizinsoziologie. Dort wird oft die gleiche Thematik untersucht, jedoch aus unterschiedlichen wissenschaftlichen Perspektiven. Andererseits gibt es Public-Health-Forschungsfelder, die meist nicht als eigene Disziplinen ausgewiesen werden, die aber die interdisziplinäre Arbeitsweise von Public Health sehr deutlich aufzeigen. Ein Beispiel hierfür ist die Gesundheitssystem- und Versorgungsforschung. Sie untersucht die Struktur, Leistungsfähigkeit und Wirksamkeit von Angeboten in den Gesundheitssystemen. Erreichbarkeit, Zugang und Nutzen von Angeboten sowie deren Kosten-Nutzen-Verhältnis spielen dabei eine bedeutende Rolle. Hierzu bedient sie sich u. a. verschiedener Methoden und Ansätze der Epidemiologie, der empirischen Sozialforschung, der Gesundheitspolitik, der Managementund Organisationsforschung sowie der Ethik.

Die vorliegende Liste kann keinen Anspruch auf Vollständigkeit erheben. So lässt sich auch argumentieren, dass weitere Fächer wie z.B. die Sportmedizin zu Public Health gehören, da sie sich im Bereich der Prävention und Gesundheitsförderung (oft in Zusammenarbeit mit der Gesundheitspsychologie und -pädagogik oder der Präventivmedizin) engagieren. Andererseits führen nicht alle Versuche einer Zusammenarbeit zwischen medizinisch-naturwissenschaftlichen Fächern und Public Health stets zu Ergebnissen, die aus Public-Health-Sicht unmittelbar relevant sind. Kapitel 1.7 diskutiert dies am Beispiel der Public Health Genomics. 


\subsection{Ansatzpunkte der Prävention}

Prävention bedeutet im wörtlichen Sinne, einer Krankheit „zuvorzukommen“ (von lat. praevenire). Um dies zu erreichen, kann Prävention an verschiedenen Punkten ansetzen:

- Auf dem als Kontinuum vorgestellten Weg von Gesundheit über Krankheit zum Tod: Primär-, Sekundär- und Tertiärprävention (vgl. a. Kap. 4.3)

- Am Individuum oder seiner Umwelt: Verhaltens- und Verhältnisprävention (s. Kap. 4.2)

- Auf Bevölkerungsebene oder bei (Hoch-)Risikogruppen

- Bei Zielgruppen, die nach ihren Krankheitsrisiken oder nach ihren Verhaltensmustern definiert werden

Ziel von Prävention ist dabei stets die Verbesserung der Gesundheit der Bevölkerung. Der folgende Abschnitt erläutert die Begriffe Primär-, Sekundär- und Tertiärprävention. Leider wird diese Terminologie nicht immer einheitlich genutzt. Zudem gibt es Übergänge zwischen den beschriebenen Formen (s.u.).

Primärprävention hat zum Ziel, das Auftreten von Gesundheitsschäden, Neuerkrankungen und Todesfällen in der Bevölkerung zu vermeiden oder zumindest die Wahrscheinlichkeit zu senken, dass die betreffenden Schädigungen oder Krankheiten auftreten. Klassische Beispiele für Primärprävention sind Maßnahmen zum Nichtraucherschutz (z.B. durch Rauchverbote in Gaststätten und öffentlichen Räumen) oder zum Anheben des "Einstiegsalters" beim Rauchen (z. B. durch die Besteuerung von Tabakprodukten, um eine finanzielle Barriere zu errichten). Auch Impfungen, wie etwa die Masernimpfung bei Kindern, gehören zur Primärprävention.

Sekundärprävention zielt darauf $a b$, klinisch noch unauffällige Frühformen von Erkrankungen zu erkennen und dadurch rechtzeitig zu behandeln, sodass die Erkrankung nicht fortschreitet oder sogar geheilt werden kann. Ein klassisches Beispiel hierfür ist das bevölkerungsweite Screening zur Früherkennung von bestimmten Krankheiten wie Brust- oder Darmkrebs (s. Kap. 4.5).

Ziel der Tertiärprävention ist es, die Verschlimmerung einer bereits manifesten Erkrankung zu verhindern oder den Vorgang zu verlangsamen. Weitere mögliche Ziele sind die Verbesserung der Lebensqualität oder der sozialen Funktionsfähigkeit. Ein typisches Beispiel hierfür sind Rehabilitationsmaßnahmen nach Eintritt einer schweren Herz-Kreislauf- oder Krebserkrankung.

Einige präventive Maßnahmen können jedoch auch Aspekte von Primär-, Sekundärund Tertiärprävention beinhalten. So lassen sich beispielsweise viele Maßnahmen der Gesundheitsberatung mehr als einem der drei genannten Ansatzpunkte zuordnen. Eine Ernährungsberatung kann bei gesunden Menschen das Ziel haben, das Auftreten eines Diabetes mellitus Typ 2 von vornherein zu verhindern. Bei Menschen mit mäßig erhöhtem Blutzuckerspiegel soll durch Ernährungsberatung das Auftreten einer klinischen Symptomatik vermieden werden. Schließlich soll sie bei manifesten Diabetikerlnnen helfen, das Risiko von Komplikationen zu senken.

Es gibt unterschiedliche Meinungen darüber, ob kurative medizinische Maßnahmen auch als Sekundär- oder Tertiärprävention bezeichnet werden können. Ein Beispiel dafür ist die Gabe von antiretroviralen Medikamenten bei HIV-positiven Menschen. Sind 
bei den Betroffenen schon Symptome aufgetreten, kann die Medikation dazu dienen, eine Verschlimmerung der Erkrankung sowie das Auftreten von durch die Immunschwäche bedingten Folgeerkrankungen zu verhindern. Die Gabe antiretroviraler Medikamente könnte hier somit als Tertiärprävention angesehen werden kann. Bei klinisch noch unauffälligen Menschen mit positivem HIV-Test kann die Medikation das Auftreten von Symptomen verhindern. Hier könnte die Maßnahme also als Sekundärprävention interpretiert werden. Da eine antiretrovirale Therapie die Viruslast im Blut der Behandelten erheblich senken kann, sodass sich die Ansteckungsgefahr für ihre Sexualpartner verringert, handelt es sich auch um eine primärpräventive Maßnahme.

\subsection{Public-Health-Ethik}

Die Ethik ist eine angewandte Disziplin der Philosophie, die es sich zur Aufgabe macht, in verschiedenen Lebensbereichen Kriterien und Normen für gutes und richtiges menschliches Handeln zu entwickeln. Als Medizinethik bezeichnet man den Teilbereich der Ethik, der sich mit dem Handeln der verschiedenen Akteure in der medizinischen Versorgung, Pflege und Forschung beschäftigt. Ethische Fragen stellen sich in der Medizin immer wieder bei der Abwägung von Risiken oder Kosten bestimmter Diagnostiken und Therapien im Verhältnis zu ihrem Nutzen, aber auch im Zusammenhang mit dem Schutz nicht-einwilligungsfähiger Personen (z. B. Demenzkranke, Kinder oder Bewusstlose) oder bei der Planung und Durchführung klinischer Studien (s. Kap. 2.1.6). Besonders kontrovers diskutierte medizinethische Themen sind Sterbehilfe und Beihilfe zum Suizid, die z. B. in Deutschland und der Schweiz sehr unterschiedlich gehandhabt werden.

Die Public-Health-Ethik ist ein relativ neues Anwendungsgebiet der Ethik, das sich mit ethischen Fragen im Bereich der öffentlichen Gesundheitspflege beschäftigt. Solche Fragestellungen können z. B. bei der Durchführung von Überwachungs- und Kontrollmaßnahmen im Rahmen von Infektionskrankheiten ebenso auftreten wie bei der Durchführung von Screening-Programmen oder bei dem Erlass von Verboten (etwa des Rauchens in öffentlichen Räumen). Während in der Medizinethik die Arzt-Patient-Beziehung im Mittelpunkt steht, ist es in der Public-Health-Ethik das Verhältnis zwischen den staatlichen und nicht-staatlichen Institutionen einerseits und den BürgerInnen andererseits.

Ethik leitet ihre Prinzipien aus theoretischen Ansätzen ab. Eine der grundlegenden ethischen Theorien ist der Utilitarismus. Handlungen werden hier im Hinblick auf ihrer Konsequenzen bewertet, und zwar unter dem Gesichtspunkt der Steigerung des allgemeinen Wohlergehens. Dieser Ansatz ist dem Denken im Bereich Public Health sehr nahe. Die Daten, auf die man sich bei der Abwägung von Nutzen, Schaden und Kosten bestimmter Maßnahmen beruft, stammen in der Regel aus epidemiologischen und ökonomischen Studien (s. Kap. 2.1.5 und 2.5.1). Ein problematischer Aspekt des Utilitarismus ist, dass hier nur das Wohlergehen der Mehrheit berücksichtigt wird. Dem Utilitarismus wird deshalb das Konzept der Menschenrechte gegenübergestellt. Viele der 30 Artikel der Allgemeinen Erklärung der Menschenrechte, die die UN-Generalversammlung im Jahr 1948 verabschiedete, sind für Public Health relevant. Hierzu gehören z. B. der Anspruch auf den Schutz vor Diskriminierung, das Recht auf Fürsorge und Gesundheit, das Recht auf Bildung und das Recht, an der Gestaltung der öffentlichen Angelegenheiten seines Landes mitzuwirken. Erwähnt sind aber auch Pflichten gegenüber der Gesellschaft (s. Internetressourcen). 
Von besonderer Bedeutung im Hinblick auf einen Berufskodex sind die folgenden sechs wichtigen Prinzipien der Medizin- und der Public-Health-Ethik, die sich in einigen Bereichen überschneiden (s. Tab. 1.1).

Tab. 1.1: Übersicht über die wichtigsten ethischen Prinzipien im Bereich der Medizinethik und der Public Health Ethik.

Medizin Public Health

Autonomie (Respect for autonomy)

Jede Person ist frei in ihren Entscheidungen.

Bei medizinischen Maßnahmen oder der

Teilnahme an einer Studie muss die

informierte Zustimmung (Informed Consent)

der betroffenen Personen vorliegen.

Fürsorge (Beneficence und Non-malefi-

cence)

Schädliche oder riskoreiche Eingriffe und

Maßnahmen sollen vermieden werden.

Durch die Maßnahme/Studie wird das Wohl

der PatientInnen oder Studienteilnehmerln-

nen gefördert.

Gerechtigkeit (Justice)

Das Prinzip der Gerechtigkeit fordert eine faire Verteilung von Gesundheitsleistungen, Risiken und Nutzen in der klinischen Forschung.
Gegenseitige Abhängigkeit (Interdependence)

Das Handeln einer Person betrifft nicht nur sie selbst, sondern auch andere Personen. Jede Person ist auch von den Aktionen anderer betroffen.

Mitwirkung (Participation)

Public-Health-Maßnahmen werden unter Mitsprache und mit dem Einverständnis der betroffenen Bevölkerung geplant und durchgeführt.

\section{Wissenschaftliche Abstützung}

(Scientific evidence)

Entscheidungen über Public-Health-Maßnahmen sollen aufgrund von wissenschaftlichen Daten und nicht auf der Basis von Annahmen und Meinungen erfolgen.

Das Prinzip der Autonomie: Bei allen Maßnahmen im Bereich der Medizin und in Public Health muss die Entscheidungsfreiheit der betroffenen Menschen respektiert werden. Auch Public-Health-Maßnahmen bedürfen daher der informierten Zustimmung (Informed Consent, s. a. Kap. 2.1.6), z. B. im Zusammenhang mit Impfungen oder Screening-Untersuchungen.

Das Prinzip der gegenseitigen Abhängigkeit: Das Prinzip der Autonomie wird durch das Prinzip der gegenseitigen Abhängigkeit ergänzt und relativiert. Es besagt, dass das Handeln eines Einzelnen in der Regel auch andere Menschen betrifft. Eine mit HIV infizierte Person muss daher andere durch den Gebrauch von Kondomen vor einer Ansteckung schützen. Durch die Impfung des Krankenhauspersonals gegen die Virusgrippe (Influenza) können HochrisikopatientInnen vor Ansteckung geschützt werden (s.a. Kap 8.4.1). Ein Rauchverbot in öffentlichen Räumen ist u.a. aufgrund der schädlichen Wirkung des Passivrauchens (s.a. Kap. 5.2.3) gerechtfertigt.

Das Prinzip der Fürsorge: Das Prinzip der Fürsorge beinhaltet die Verpflichtung, den Menschen Gutes zu tun und Schaden zu vermeiden. In Kombination mit dem Menschenrecht auf Gesundheit und Fürsorge lässt sich hieraus eine Verpflichtung des Staates ableiten, sich im Bereich Public Health zu engagieren. Hierzu gehört, dass die 
Lebensbedingungen der Menschen so gestaltet werden sollen, dass die einzelnen BürgerInnen Verantwortung für ihre Gesundheit übernehmen können. Dies ist in vielen Ländern bislang nicht der Fall (s. Kap. 9.2).

Das Prinzip der Mitwirkung: Dem Prinzip der Fürsorge steht das Prinzip der Mitwirkung gegenüber: Public-Health-Maßnahmen sollen stets unter Einbezug der betroffenen Bevölkerung geplant und umgesetzt werden. Handlungen, die gegen den Willen oder ohne die informierte Zustimmung der Betroffenen zu deren Wohl durchgesetzt werden (Paternalismus), widersprechen diesem Prinzip.

Das Prinzip der Gerechtigkeit: Gerechtigkeit wird in der Medizinethik vorwiegend als distributive Gerechtigkeit verstanden. Dies bedeutet, dass die Leistungen des Gesundheitssystems allen offen stehen und die Kosten fair auf die Mitglieder der Gesellschaft verteilt werden sollen (s. a. Kap. 3.1.3). Im Public-Health-Kontext leitet sich daraus die Verpflichtung ab, sozio-ökonomisch bedingte Ungleichheiten im Gesundheitszustand der Bevölkerung (s. Kap. 1.3.2) zu verringern. Es muss dabei darauf geachtet werden, dass sich die bestehenden Ungleichheiten nicht durch Public-Health-Maßnahmen, die vor allem von sozial Bessergestellten in Anspruch genommen werden, weiter vergrößern. Zum Prinzip der Gerechtigkeit gehört auch der Schutz vor Diskriminierung und Stigmatisierung.

Das Prinzip der wissenschaftlichen Abstützung: Die Vor- und Nachteile von PublicHealth-Maßnahmen sollen schließlich nicht von gesellschaftlichen Moralvorstellungen, Meinungen und Annahmen geleitet, sondern auf der Basis guter wissenschaftlichen Daten diskutiert werden (Prinzip der wissenschaftlichen Abstützung). Dieses Prinzip gilt sinngemäß auch für die Medizinethik, wird aber im Public-Health-Kontext besonders betont, weil hier oft gesunde Menschen betroffen sind.

Als erste Formulierung eines ethischen Berufskodex gilt der Eid des Hippokrates, benannt nach dem griechischen Arzt Hippokrates von Kós (um 460 bis 370 v. Chr.). Im Jahr 1947 wurde der Nürnberger Kodex als Reaktion auf die während der Zeit des Nationalsozialismus im Namen der medizinischen Forschung begangenen Verbrechen verfasst. Er enthält ethische Richtlinien für die Durchführung von Experimenten am Menschen. Der Weltärztebund verabschiedete 1964 die Deklaration von Helsinki zu ethischen Grundsätzen für die medizinische Forschung am Menschen. Sie wurde seither mehrmals revidiert und dient heute den Ethikkommissionen als Grundlage für die Beurteilung klinischer Studien (s. a. Kap. 2.1.6). Im Bereich der Medizin haben ethische Diskussionen also anders als in Public Health schon eine lange Tradition. Die in den USA entwickelten ausführlichen Richtlinien für ethisches Handeln in Public Health (Principles for the Ethical Practice of Public Health) wurden von der American Public Health Association erst 2002 angenommen (s. Internet-Ressourcen).

\subsection{Public Health Genomics}

\section{Albrecht Jahn, Nicole Probst-Hensch}

Public Health Genomics hat zum Ziel, genombasiertes Wissen und die dazu entwickelten Technologien mit Public-Health-Forschung, Gesundheitspolitik und Gesundheitsprogrammen zu verknüpfen (s. Office of Public Health Genomics). 
Die Erkenntnis, dass genetische Faktoren bei der Entstehung und Manifestation vieler Krankheiten eine wichtige Rolle spielen, ist nicht neu. Von praktischer Bedeutung ist sie bisher vor allem bei monogenetischen Erkrankungen, wie z. B. der Phenylketonurie (PKU), bei der ein Enzymdefekt, verursacht durch eine Mutation in einem einzelnen Gen, dazu führt, dass sich die Aminosäure Phenylalanin im Körper der Betroffenen anreichert. Hierdurch kommt es zu einer schweren geistigen Entwicklungsstörung. Die frühzeitige Erkennung einer PKU über einen Bluttest bei Neugeborenen bietet die Möglichkeit, die Manifestation der Erkrankung durch eine phenylalaninarme Diät zu verhindern. Der Bluttest wird u. a. in Deutschland und der Schweiz im Rahmen des Neugeborenen-Screenings flächendeckend durchgeführt und gilt als ein Paradebeispiel für eine sinnvolle Screening-Maßnahme. (s. Kap. 4.5.4). Seit einiger Zeit kann die PKU auch bereits vorgeburtlich mit Hilfe eines Gentests diagnostiziert werden. An solche genetischen Tests werden im Hinblick auf Sensitivität und Spezifität (s. Kap. 2.3.7) die gleichen Anforderungen wie an andere Tests im Bereich der klinischen Diagnostik und des Screenings gestellt.

Anders als die Genetik, die - wie im Beispiel der PKU - die Funktion einzelner Gene oder Gen-Kombinationen untersucht, befassen sich die Genomics mit dem gesamten Genom eines Organismus, um die Funktionen und Interaktionen von Genen und deren Produkten besser zu verstehen. Mit der Entschlüsselung des menschlichen Genoms im Jahr 2001 waren zunächst hochgesteckte Erwartungen im Hinblick auf medizinische Anwendungen der neuen Erkenntnisse verbunden. Diese Hoffnung hat sich insbesondere in Bezug auf die primäre und sekundäre Prävention nicht übertragbarer und chronischer Krankheiten bislang noch nicht in dem erwarteten Ausmaß erfüllt.

Derzeit versucht man, individuelle, auf genetischen Parametern beruhende, maßgeschneiderte Behandlungs- und Präventionsmaßnahmen zu entwickeln. Vertreter einer solchen „personalisierten Medizin“ sprechen davon, dass sich in Zukunft auch das Fach Public Health weg von den klassischen, populationsbezogenen Konzepten, hin zu einer individualisierten Vorsorge und einer "Personalised Public Health" entwickeln wird. Durch die Kenntnis ihrer genetischen Ausstattung könnten dann z. B. alle Menschen innerhalb einer Bevölkerung in die Lage versetzt werden, die für sie jeweils richtige Ernährungsweise zu wählen. Zur Analyse ihres Genotyps würden zuvor Testverfahren wie die Genomsequenzierung, DNA-Mikroarrays und die Schlüsseltechniken der Proteomik eingesetzt.

Diese Zukunftsvision berücksichtigt jedoch nicht, dass insbesondere im Bereich der chronischen, nichtübertragbaren Krankheiten (z. B. der Herz-Kreislauf-Erkrankungen [s. Kap. 7.1] und der bösartigen Tumore [s. Kap. 7.2]) nur wenige Krankheitsbilder durch ein einzelnes krankhaftes Gen verursacht werden. An der Entstehung der meisten Krankheiten sind mehrere Gene beteiligt. Darüber hinaus spielen hier auch komplexe Interaktionen zwischen Genen und Umwelt eine große Rolle. So können z. B. Faktoren, die prä- und perinatal auf den Menschen einwirken, an der Krankheitsentstehung (z. B. von Adipositas und Diabetes mellitus) mitwirken. Solche epigenetischen und non-genomischen $^{3}$ Phänomene sind in der Lage, physiologische Abläufe im Körper eines Menschen bleibend zu beeinflussen. Als epigenetische Veränderungen bezeichnet man Veränderungen von Zelleigenschaften, die nicht oder nicht permanent in der DNA festgelegt

\footnotetext{
3 Non-genomisch: nicht auf genetischem Wege, nicht die Erbanlagen betreffend
} 
sind, jedoch trotzdem auf die Tochterzellen vererbt werden. Durch diese Veränderungen werden Chromosomenabschnitte oder ganze Chromosomen in ihrer Aktivität beeinflusst. Die Reihenfolge der Nukleotid-Bausteine in der DNA ändert sich hierdurch jedoch nicht.

In der Vergangenheit hat sich die Untersuchung des Zusammenhangs zwischen genetischer Variabilität und Krankheitsrisiko vor allem auf Mutationen und Polymorphismen ${ }^{4}$ in spezifischen Genen beschränkt, von denen man annahm, sie hätten eine biologische Bedeutung (Kandidatengen-Ansatz). Da inzwischen die DNA von zahlreichen Menschen mit unterschiedlichem ethnischem Hintergrund genotypisiert und sequenziert werden konnte, kennt man heute eine Vielzahl von einzelnen Abweichungen in der Basenabfolge der DNA (= Single Nucleotide Polymorphisms [SNP]) und weiß, wie sie zueinander in Beziehung stehen. So können heute mit Hilfe von Genchips mehr als 1 Million Genvarianten, die über die ganze DNA einer einzelnen Person verteilt sind, schnell und kostengünstig bestimmt oder mit großer Wahrscheinlichkeit vorhergesagt werden. Im Rahmen von genomweiten Fall-Kontroll- oder Assoziationsstudien (Genome Wide Associations - GWA) wird so ohne vorher vorhandene Hypothesen nach typischen Mustern in Genen und Chromosomenregionen im menschlichen Erbgut gesucht, die mit der Entstehung einer spezifischen Krankheit in Beziehung stehen könnten. Bislang hat man schon eine Vielzahl von SNPs gefunden, die auf ein erhöhtes Krankheitsrisiko hindeuteten. Während diese neuen Erkenntnisse für das künftige Verständnis der Krankheitsentstehung, für die Identifikationen kausaler Risikofaktoren und für die Entwicklung neuartiger Medikamente möglicherweise bedeutsam sind, ließen sich aus der Kenntnis dieser genetischen Muster jedoch noch keine sinnvollen Tests für die Vorhersage eines Erkrankungsrisikos oder für die Früherkennung einzelner Krankheiten (vgl. Box 4.5.2) ableiten. Zudem blieben in den bisher durchgeführten genomweiten Studien Umwelt- und Lebensstilfaktoren weitgehend unberücksichtigt. Da die zentrale Bedeutung dieser modifizierbaren Faktoren bei vielen chronischen Krankheiten seit langem bekannt ist und ihre Häufigkeit infolge der demografischen Veränderungen (s. Kap. 2.2) und der zunehmenden Verwestlichung von Lebensstil und Umwelt in den letzten Jahrzehnten stark zugenommen hat, könnten Gen-Umwelt-Interaktionsanalysen hier weitere Erkenntnisse bringen.

Obwohl die bisher identifizierten Genvarianten die Kriterien für einen effizienten Screening-Test bei weitem nicht erfüllen, werden seit einiger Zeit v. a. über das Internet zahlreiche Tests erfolgreich vermarktet, die ein personalisiertes Präventions- und Diätkonzept versprechen. Brauchbare, neue prädiktive ${ }^{5}$ und diagnostische Tests, die auf dem Boden von Genom-Analysen entwickelt wurden, sind derzeit v. a. bei monogenetischen Erkrankungen in Sicht. Da solche Erkrankungen jedoch nur einen geringen Anteil der gesamten Krankheitslast (Burden of Disease; s. Kap. 9.1.2) ausmachen, sind sie für Public Health lediglich von untergeordneter Bedeutung. Denkbar wäre jedoch, dass sich in Zukunft mit Hilfe solcher Methoden Subpopulationen identifiziert ließen, die ein besonders hohes Krankheitsrisiko aufweisen. Dies würde man jedoch passender mit dem Begriff der „stratifizierten Medizin“ bezeichnen. Ob solche Maßnahmen zu einer

4 Polymorphismus: das Auftreten einer oder mehrerer Genvarianten innerhalb einer Population

5 Prädiktiver genetischer Test: Gentest bei einer Person, die zum Zeitpunkt der Untersuchung noch keine Symptome einer Erkrankung zeigt 
effektiveren und kostengünstigeren Prävention bzw. Therapie beitragen würden, ist heute noch zweifelhaft.

Wie auf dem UN-Gipfel zu nichtübertragbaren Erkrankungen (2011) hervorgehoben wurde, kann ein wesentlicher Teil der vorzeitigen Krankheits- und Todesfälle aufgrund von Herz-Kreislauf-Erkrankungen, Erkrankungen der Luftwege, Krebs und Diabetes mellitus durch eine Reduktion weniger Risikofaktoren (Rauchen, übermäßiger Alkoholgenuss, fehlende Bewegung und ungesunde Ernährung) verhindert oder zumindest in ein höheres Alter verschoben werden. Die UN-Mitgliedsstaaten sind daher aufgefordert, entsprechende Maßnahmen durch gesetzliche Regulationen und Vereinbarungen mit der Nahrungsmittel- und Getränkeindustrie umzusetzen. Im Vergleich zu den hierdurch möglichen positiven Einflüssen auf die Krankheitslast weltweit leistet Public Health Genomics derzeit (noch) keinen wesentlichen Beitrag zu einem Gesundheitsgewinn auf der Bevölkerungsebene.

\section{Internet-Ressourcen}

Auf unserer Lehrbuch-Homepage (www.public-health-kompakt.de) finden Sie Links zu den hier verwendeten Quellen, zu weiterführender Literatur sowie zu anderen themenrelevanten Internet-Ressourcen. 
